\title{
Measurement of Vortex Beam Phase by Electron Holography
}

\author{
Ken Harada ${ }^{1}$, Kodai Niitsu ${ }^{1}$, Keiko Shimada ${ }^{1}$, Yoshimasa A. Ono ${ }^{1}$, and Daisuke Shindo ${ }^{1,2}$ \\ 1. CEMS, RIKEN (The Institute of Physical and Chemical Research), Hatoyama, Saitama, Japan \\ 2. IMRAM, Tohoku University, Sendai, Miyagi, Japan
}

Electron vortex beams are considered as new probes for next-generation electron beam machines, especially for transmission electron microscopes, because the vortex beams carry intrinsic orbital angular momentum, leading to unprecedented measurement capabilities $[1,2]$. To realize a practical measurement system with vortex beams, a few experiments were attempted to measure intensity distributions or energy-loss distributions [3]. In previous studies, we succeeded in controlling vortex beams in the reciprocal space by using special opening shapes of the fork-shaped gratings [4]. In the present study, electron holography techniques were used in the optical system to measure phases of vortex beams. The principle idea is the same as that of Gabor's original in-line holography. Electron beams passed around the fork-shaped grating were used as the reference waves and defocused images from the diffraction pattern were recorded as holograms of the vortex beams.

Figure 1(a) shows a transmission electron microscope (TEM) image of the fork-shaped grating of a $\mathrm{Si}_{3} \mathrm{~N}_{4}$-membrane of $100 \mathrm{~nm}$ thickness fabricated by a focused ion beam machine (NB-5000, Hitachi High-Technologies Corp.). Opening size of the grating was about $5 \mu \mathrm{m}$ in diameter and average lattice constant was about $300 \mathrm{~nm}$. The vortex beams generated from the grating were observed with a 300-kV field-emission TEM (HF-3300S, Hitachi High-Technologies Corp.) in the reciprocal space (see Fig. 1(b) and (c)). The optical system of the microscope was modified to observe small angle electron diffraction (SmAED) patterns with the camera length of about $800 \mathrm{~m}$, and the first and second diffraction spots under infocus and defocused conditions were recorded by a slow-scan charge-coupled device camera (UltraScan ${ }^{\circledR}$ 4000, Gatan Co.). Interference fringes around the circular spots in Fig. 1(c) indicate that they are electron holograms.

Figure 2 shows a reconstructed image of amplitude distribution ((a) and (c)), and phase distribution ((b) and (d)), respectively. Both amplitude and phase images are shown in clear circular shapes. Figures 2(a) and (b) are reconstructed from the first-ordered diffraction spot in Fig. 1(c), and Figs. 2(c) and (d) are from the second-ordered diffraction spot. Reconstructed phase images have circular, vortex shape, such as clockwise vortex on the right side of the image and counterclockwise vortex on the left side. This is because that the hologram (Fig. 1(c)) was interfered by using a spherical reference wave from the zeroordered diffraction spot. Two beam interference between a plane-wave-type helical wave and a spherical wave generates vortex-shape interference fringes with the azimuthal direction depending on the shape of the spherical wave, resulting in the convex shape for the under-focused condition and the concave shape for the over-focused condition. We have confirmed that the vortex-rotation direction was interchanged when the defocusing condition was interchanged.

Figure 3 shows a composite image of amplitude and phase reconstructed images to clarify relations among the four vortex beams. Four vortex beams generated by the fork-shaped grating are clearly reconstructed by the holography technique.

These results indicate that phase distribution of the vortex beams can be observed by using electron 
holography. We believe this developed technique will be applied to a practical vortex beam measurement system.

\section{References:}

[1] M. Uchida and A Tonomura, Nature 464 (2010) p. 737.

[2] B. J. McMorran et al., Science 331 (2011) p. 192.

[3] P. Schattschneider et al., Ultramicroscopy 136 (2014) p. 81.

[4] K. Harada et al., Microsc. Microanal. 20 (2014) p. 274; ibid. 21 (2015) p. 669.
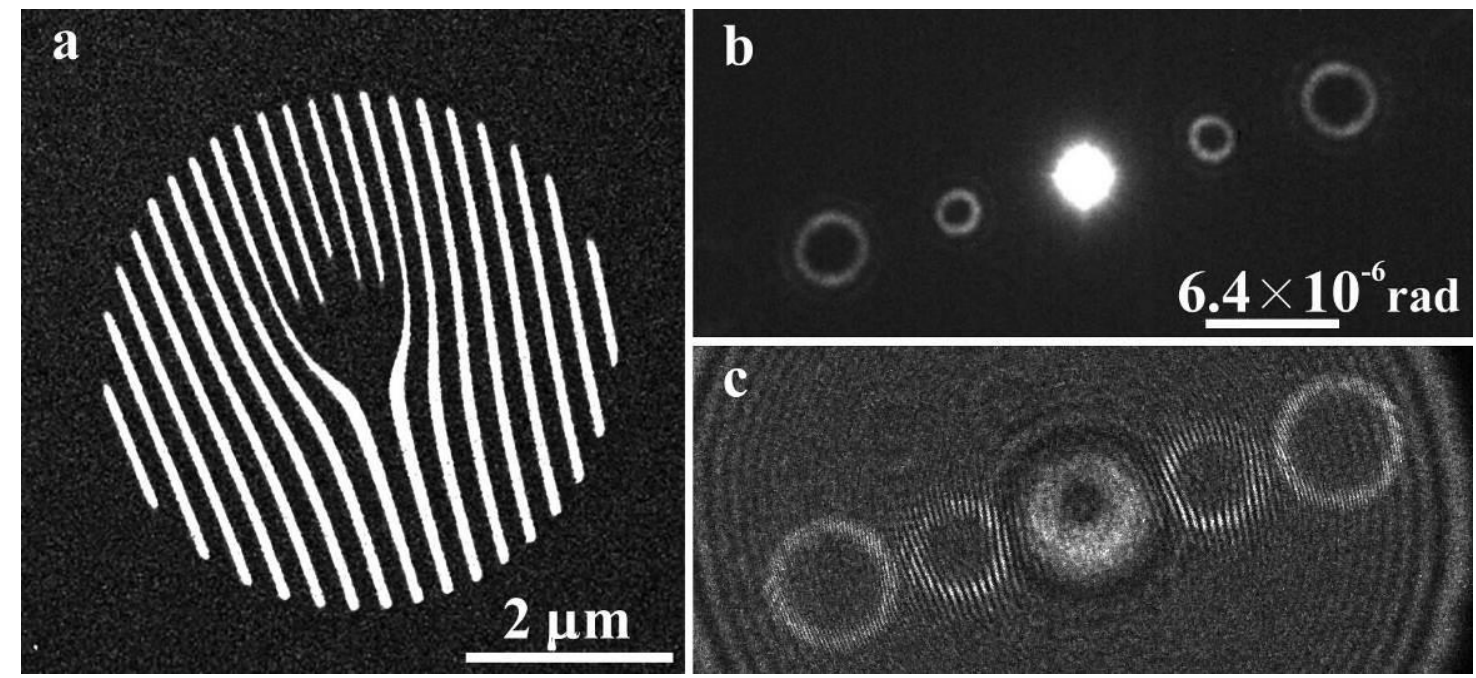

Figure 1. (a) Fork-shaped grating of SiN membrane, (b) SmAED pattern, and (c) Defocused SmAED pattern as electron hologram.

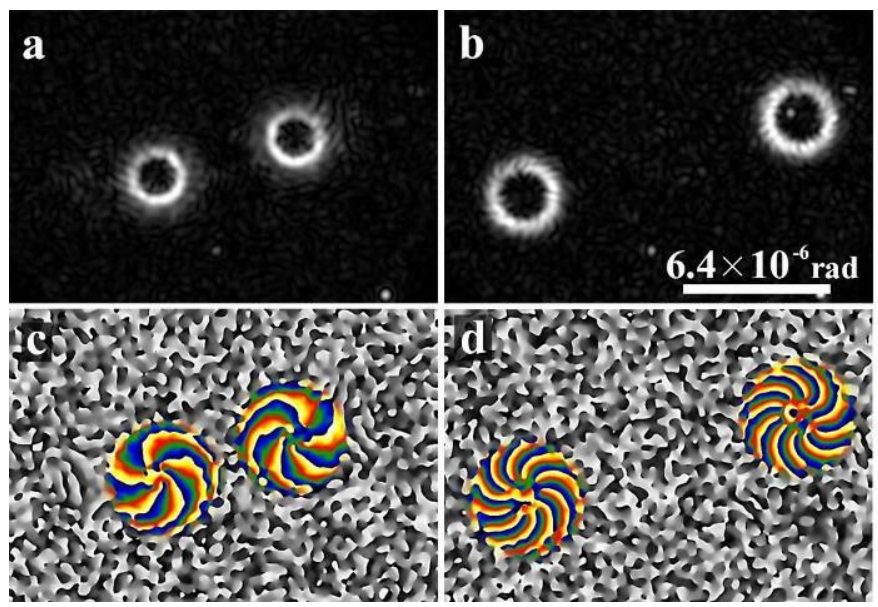

Figure 2

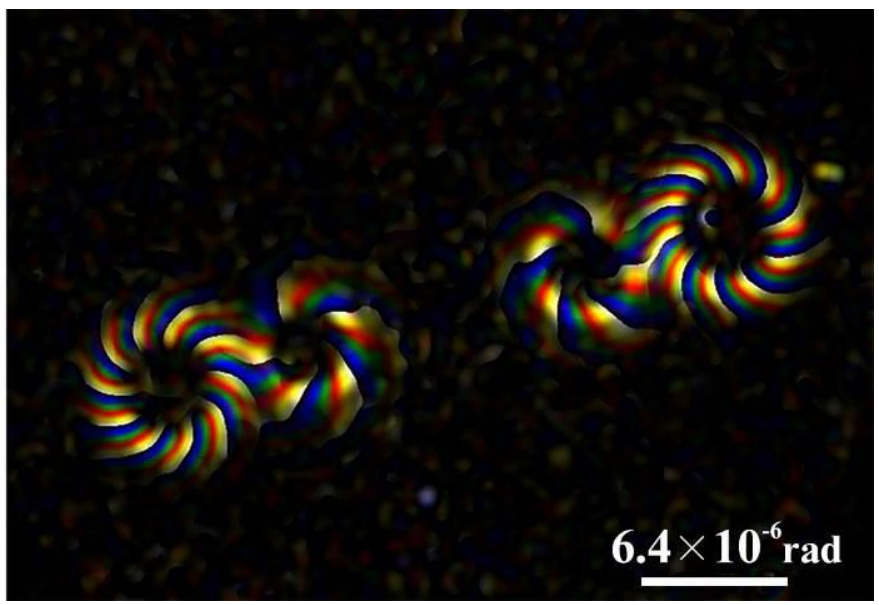

Figure 3

Figure 2. Reconstructed images from Fig. 1(c). (a) and (b) are amplitude and phase images of the first SmAED spot, (c) and (d) are amplitude and phase images of the second spot.

Figure 3. Composite image of amplitude and phase images of Fig. 2. 